\title{
In silico genetic robustness analysis of microRNA secondary structures: potential evidence of congruent evolution in microRNA Wenjie Shu ${ }^{\dagger 1,2}$, Xiaochen Bo* ${ }^{*} 1$, Ming Ni ${ }^{1}$, Zhiqiang Zheng ${ }^{2}$ and Shengqi Wang*1
}

\begin{abstract}
Address: ${ }^{1}$ Beijing Institute of Radiation Medicine, Beijing 100850, China and ${ }^{2}$ College of Electro-Mechanic and Automation, National University of Defense Technology, Changsha, Hunan 410073, China

Email: Wenjie Shu - shuwj@bmi.ac.cn; Xiaochen Bo* - boxc@bmi.ac.cn; Ming Ni - ni.ming@163.com; Zhiqiang Zheng - xyzheng@sohu.com; Shengqi Wang* - sqwang@bmi.ac.cn

* Corresponding authors †Equal contributors
\end{abstract}

Published: 13 November 2007

BMC Evolutionary Biology 2007, 7:223 doi:10.1/86/147/-2148-7-223
Received: 5 June 2007

Accepted: 13 November 2007

This article is available from: http://www.biomedcentral.com/I47I-2/48/7/223

(c) 2007 Shu et al; licensee BioMed Central Ltd.

This is an Open Access article distributed under the terms of the Creative Commons Attribution License (http://creativecommons.org/licenses/by/2.0), which permits unrestricted use, distribution, and reproduction in any medium, provided the original work is properly cited.

\begin{abstract}
Background: Robustness is a fundamental property of biological systems and is defined as the ability to maintain stable functioning in the face of various perturbations. Understanding how robustness has evolved has become one of the most attractive areas of research for evolutionary biologists, as it is still unclear whether genetic robustness evolved as a direct consequence of natural selection, as an intrinsic property of adaptations, or as congruent correlate of environment robustness. Recent studies have demonstrated that the stem-loop structures of microRNA (miRNA) are tolerant to some structural changes and show thermodynamic stability. We therefore hypothesize that genetic robustness may evolve as a correlated side effect of the evolution for environmental robustness.
\end{abstract}

Results: We examine the robustness of I,082 miRNA genes covering six species. Our data suggest the stem-loop structures of miRNA precursors exhibit a significantly higher level of genetic robustness, which goes beyond the intrinsic robustness of the stem-loop structure and is not a byproduct of the base composition bias. Furthermore, we demonstrate that the phenotype of miRNA buffers against genetic perturbations, and at the same time is also insensitive to environmental perturbations.

Conclusion: The results suggest that the increased robustness of miRNA stem-loops may result from congruent evolution for environment robustness. Potential applications of our findings are also discussed.

\section{Background}

Robustness, a fundamental and ubiquitously observed phenomenon in biological systems, is defined as the ability to maintain stable functioning in the face of various perturbations, and is characterized as genetic or environmental robustness, depending on whether the perturba- tions are inheritable or not [1]. Genetic robustness describes insensitivity of a phenotype facing genetic mutations, and the insensitivity to environmental factors is called environmental robustness. Phenotype robustness appears at various levels of biological systems, including gene expression, protein folding, metabolic flux, physio- 
logical homeostasis, development, and even organism fitness [2]. It is consequently not surprising that biologists have a long-standing interest in robustness, going back to Fisher's work on dominance [3-5], and to Waddington's developmental canalization research [6,7]. Hiroaki Kitano argues that the requirements for robustness and evolvability are similar, since robustness facilitates evolution and evolution favours robust traits [8]. A proper understanding of the origin of robustness in biological systems will catalyze our understanding of evolution [9].

The evolution of mechanism underlying the buffering of the phenotype against genetic and environmental influences has received much theoretical and experimental attention in recent years, yet the evolutionary origin of the observed robustness remains unresolved. Whether it is a consequence of natural selection or a nonadaptive correlated side-effect of other phenotypic traits is by and large unknown. A recent review article categorized the theories addressing the evolution of genetic robustness into three main classes: adaptive, intrinsic, and congruent [2]. The most straightforward explanation for the evolution of robustness, according to the Darwinian tradition, is adaptive robustness. In this scenario, almost all mutations lead to deviations from the optimum, and robustness is favored by natural selection. High mutation rates, large populations, and asexual reproduction generally favor the evolution of robustness $[10,11]$. Genetic robustness may also evolve simply because buffering is a necessary consequence of character adaptation; that is, robustness is a nonadaptive correlated side effect of the stabilizing selection acting on other traits [12]. Additionally, because environmental perturbations often have a higher frequency and stronger impact on fitness, they will serve as the driving force; that is, genetic robustness evolves as a correlated side-effect of the evolution for environmental robustness. This is an appealing hypothesis as there is no aspect of an organism that is inherently and persistently vulnerable to genetic but not environmental perturbations [12]. Support for this theory comes from a recent computational study of RNA secondary structure by Ancel and Fontana [13], who find that RNA shapes that are robust against environmental (thermodynamic) perturbations are also robust against mutational perturbations. Simplified modeling of protein structures suggests that a similar correlation between genetic robustness and thermodynamic stability might also exist for proteins [14-16]. Further supports come from recent studies of heat-shock proteins, such as Hsp90 and GroEl, which are thought to have evolved to protect organisms from environmental and developmental perturbations, but appear to also buffer against genetic perturbation in Drosophila [17], Arabidopsis [18], and Escherichia coli [19]. However, to date, the extent to which each of these evolutionary forces contributes to the evolution of robustness remains unre- solved, partly due to the difficulty in providing evidence for robustness in natural biological systems [20].

Addressing this challenge, recent studies have resorted to bioinformatics and experimental approaches. One important effort to provide the evidence of environmental robustness has focused on the thermodynamic stability of noncoding RNA secondary structures [21-26]. Although the shuffling sequences generated in these studies rule out the bias of base composition, they do not preserve the structural phenotype of the native RNA sequences. Consequently, it cannot be determined whether the observed increased robustness goes beyond the intrinsic robustness of specific, functionally important structures. Comparing with environmental robustness, genetic robustness, on the other hand, is connected with major technical difficulties [20]. The classical approach has inferred genetic robustness from the increase in genetic variance after a major mutation or exposure to an environmental challenge during development [2], as exemplified by the measurements of vibrissae number in mice, ocelli in $D$. subobscura, and wing- and cross-vein interruptions and scutellar bristle numbers in $D$. melanogaster, which are all discussed in detail by Scharloo [27]. However, the evidence is often indirect and suffers from the lack of a natural reference genotype [20]. Experimental evolution is a more direct approach that has been applied to the study of robustness recently, which utilizes direct laboratory observation of short-term evolutionary processes, mostly in microbes $[19,28]$. Although its evolutionary potential is limited by time constraints, this approach does not suffer from a lack of control and promises exciting new data and insights for a more comprehensive theory of the evolution of genetic robustness [2].

Using a plausible background model is another strategy applied to elucidate the evolution of robustness, allowing evaluation of the significance of any greater robustness found in the wild-type (WT) [2]. Wagner and Stadler have compared the mutational stability of conserved and nonconserved elements in the secondary structure of a RNA viral genome with respect to point mutations, using nonconserved elements as the reference set [29]. Their research has demonstrated that the conserved elements show a consistently lower variability than non-conserved elements, suggesting that the virus evolves to a state of increased mutational robustness. While their data do not prove that selection that acts directly on the mutational stability of the RNA secondary structure, increases thermodynamic stability, this study do provide the first hint that "genetic canalization" (genetic robustness) can, in fact, evolve as a correlated response to selection for "environmental canalization" (thermodynamic stability), as predicted by population genetic models [1]. On the other hand, experimental studies have demonstrated that 
miRNA secondary structures are tolerant to some structural changes [30-33], with reports that miRNA precursors exhibit a significantly higher level of thermodynamic stability [21]. We therefore hypothesize that miRNA genetic robustness may evolve as a correlated side effect of the evolution for environmental robustness. To our knowledge, no systematic effort has been made to test this hypothesis in a genome-wide scale, with the exception of an experimental study on the effect of single point mutation for limited miRNAs [30-33].

Previous researches have demonstrated that miRNAs are abundant endogenous 22-nucleotide (nt) noncoding RNAs, occupying between $1-5 \%$ of the genes in any given animal genome [34]. miRNAs regulate gene expression at the post-transcriptional level for cleavage or translational repression through the binding of a minimal-recognition 'seed' sequence [35-38]. Recent comparative phylogenetic studies have revealed that conserved miRNA-binding sequences are in more than one-third of all genes, suggesting that miRNA regulation may be relevant to a large portion of cellular processes [39-44]. Mature miRNAs are cleaved from $70 \mathrm{nt}$ precursors (pre-miRNA) that fold into a stem-loop hairpin structure, through the action of Dicer endonuclease [45-47]. The miRNA stem-loop structure is conserved in evolution, and plays a crucial role throughout miRNA gene maturation processing steps $[33,46,48-50]$. Additionally, there is a selective pressure to stabilize the stem-loop structure and some structural changes are tolerated [51], which may result in the evolution of robustness. As well, the use of program packages for RNA secondary structure prediction, such as Mfold [52] and Vienna RNA package [53], facilitates genotypephenotype mapping and measurement of the structural robustness of a given miRNA stem-loop sequence, by comparing the predicted structure of this sequence with the predicted structure of all its one-mutant neighbors. Different types of reference sequences, with similar phenotypes and/or with exact or nearly exact mononucleotide and dinucleotide base composition as the real premiRNA, can be easily generated for miRNAs, allowing for careful control of the effects of secondary structure evolution. These merits make the miRNA stem-loop structure an ideal system to study the evolution of genetic robustness.

In our previous study, we have developed a method to quantitatively measure the genetic robustness of RNA secondary structure [54]. Here, we will apply this method to investigate the robustness of 1,082 miRNA genes from six different species. Our data suggest that the hairpin structures of miRNA precursors exhibit a significantly higher level of mutational robustness. Additionally, through the careful design of reference backgrounds, we show that this excess robustness goes beyond the intrinsic robustness of the stem-loop structure, and is not the byproduct of a base composition bias. Examination of the environmental robustness of real miRNA stem-loops also demonstrates that the phenotype of miRNAs buffers against genetic variations, at the same time is insensitive to environmental perturbations. These data suggest that the increase in genetic robustness may evolve as a correlated side effect of the evolution for environmental robustness.

\section{Results}

For each real pre-miRNA, the robustness $\gamma_{1}^{m}$ at threshold level $T_{1}$ is compared with that of 1,000 random sequences, and the $P$-value versus $Z$-score is showed in Figure 1(a). Figure 2(a) shows the corresponding $P$-value distribution of genetic robustness for the 1,082 miRNAs at threshold level $T_{1}$. Of the 1,082 miRNA investigated, 920 (85.0\%), 534 (49.4\%), 376 (34.8\%) miRNA genes show significant increases in robustness at FDR-controlled $P$-values of $<$ $0.05,<0.01$, and $<0.005$, respectively (Figure 3 and Table $\mathrm{S} 1$, Additional file 15). We further compare the robustness $y_{1}$ of each real pre-miRNA with that of 1,000 pseudo premiRNAs, and the $P$-value versus $Z$-score is showed in Figure $1(\mathrm{~b})$, so as to exclude the intrinsic robustness associated with the stem-loop hairpin structure. The corresponding $P$-value distribution of genetic robustness for the 1,082 miRNAs at threshold level $T_{1}$ is showed in Figure 2(a). Our results suggest 787 (72.7\%) miRNA genes of 1,082 are significantly more robust than pseudo pre-miRNAs at FDR-controlled $P$-value of $<0.05$ (Figure 3 and Table S2, Additional file 15). These data suggest that the genetics of miRNA robustness is not a byproduct of the specific stem-loop structure. Additionally, investigation of the robustness $y_{1}$ of miRNA genes within species is similar for each species individually (Table $1-2$ ).

The mononucleotide and dinucleotide frequencies of an RNA sequence, not preserved in random sequences, are crucial for the physical stability of the secondary structure $[21-23,26]$. It is consequently essential to verify that the greater robustness of real pre-miRNAs is not a byproduct of a bias in the base composition of the real pre-miRNA sequences, compared with random sequences. To this end, we generate four types of shuffled miRNAs that preserve the exact or nearly exact mononucleotide and dinucleotide base composition as the real pre-miRNA (see Methods). The robustness $\gamma_{1}^{m}$ of each real pre-miRNA is compared with that of 1,000 shuffled sequences generated by four types of shuffled methods, and the P-value versus $Z$-score is showed (Additional files 1 and 2). More 
(a)

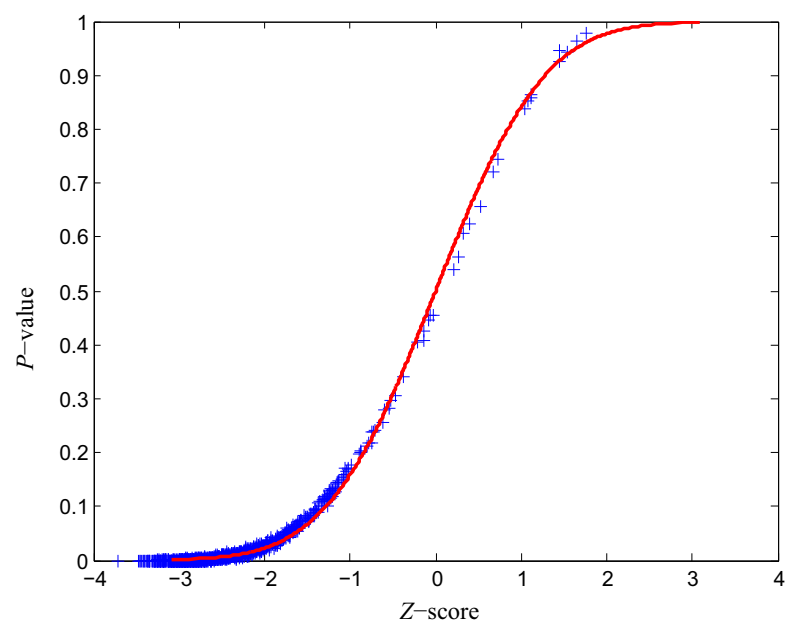

(b)

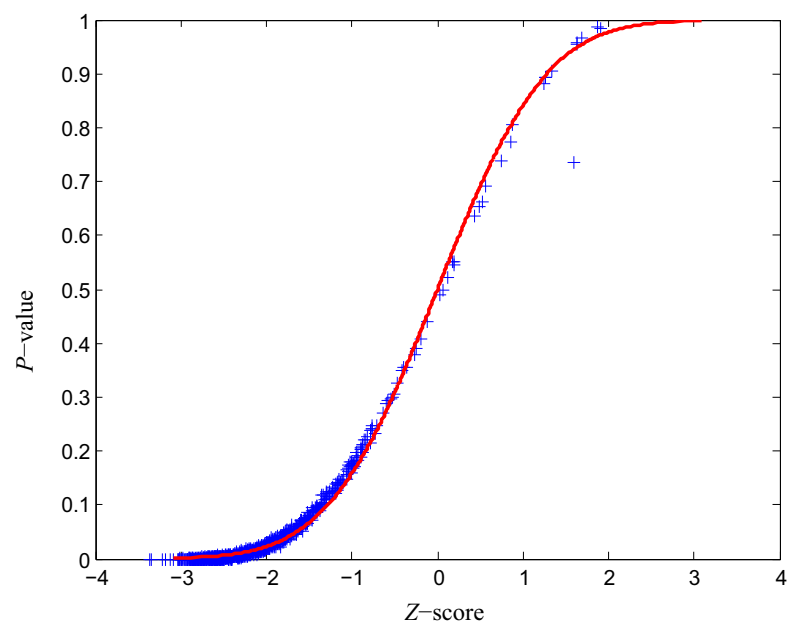

Figure I

Correlation between Z-score and P-value of genetic robustness for all the I,082 miRNAs at threshold level $T_{1}$.

For each real pre-miRNA, the robustness $\gamma_{1}^{m}$ is compared with that of 1,000 random sequences (a) and random pseudo premiRNAs (b), and the Z-score and $P$-value are computed. The curve for a normal distribution of mean 0 and a standard deviation of $\mathrm{I}$ is also displayed.

than 910 of the 1,082 (>84\% for the four types of shuffled methods) miRNA sequences have a significantly larger $\gamma_{1}^{m}$ at FDR-controlled P-value of $<0.05$, with a high proportion ( $>34 \%$ for the four types of shuffled methods) is also observed at FDR-controlled P-value of $<0.005$ (Figure 3 and Table S1, Additional file 15). To further confirm

(a)

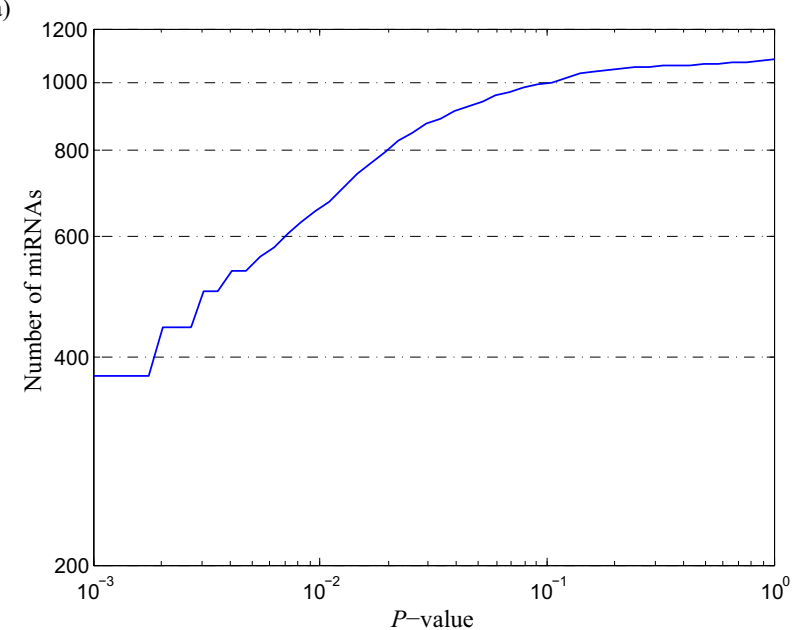

that the excess robustness goes beyond the intrinsic robustness of the stem-loop structure, we generate four types of reference sets for each miRNA, consisting of 1,000 shuffled pseudo pre-miRNAs that preserve not only the stem-loop structure, but also the exact or nearly exact mononucleotide and dinucleotide frequencies as real pre-

(b)

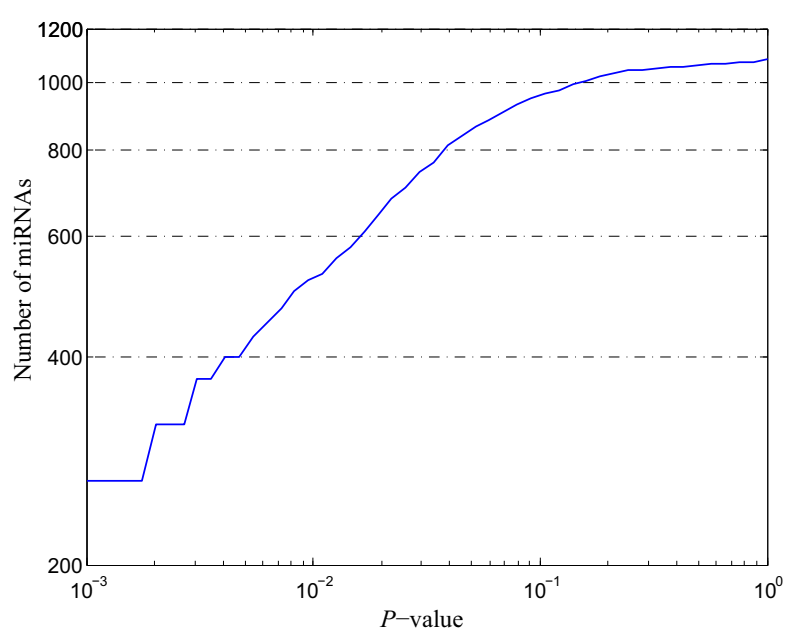

Figure 2

$P$-value distribution of genetic robustness for the I,082 miRNAs at threshold level $T_{1}$. For each real pre-miRNA, the robustness $\gamma_{1}^{m}$ is compared with that of 1,000 random sequences (a) and random pseudo pre-miRNAs (b). 


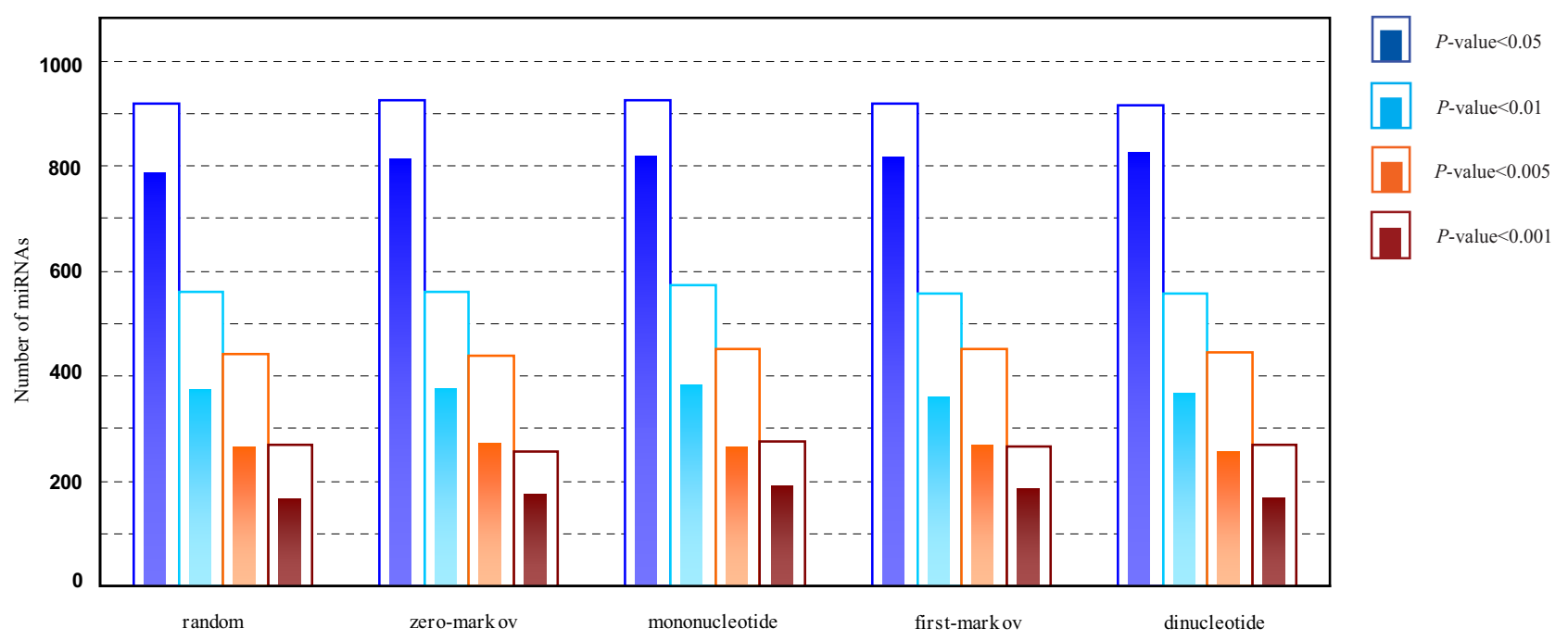

Figure 3

Number of genetically robust miRNAs with FDR-controlled $P$-values of $<0.05,0.01,0.005$, and 0.001 at threshold level $T_{1}$. 2D histogram plots of the number of miRNAs with significantly genetic robustness. Each bar is constituted with an outer hollow sub-bar and an inner solid sub-bar, which represents the number of significantly robust real premiRNAs compared with random/shuffled sequences and random/shuffled pseudo pre-miRNAs, respectively.

miRNA (see Methods). Of the 1,082 miRNA genes, over $810(>75 \%)$ miRNAs show significant more robust than shuffled pseudo pre-miRNA sequences at FDR-controlled P-value of $<0.05$ (Figure 3, Table 2, Additional files 3 and 4, and Table S2- Additional file 15), suggesting that the genetic robustness of miRNA is not a byproduct of the specific stem-loop structure and that there is no bias of the base composition on the intrinsic robustness analysis. These results also demonstrate that the different types of shuffled methods are indistinguishable (Figures 3, Additional files 1, 2, 3, 4, Tables 1, 2, and Table S1-S2, Additional file 15).

To test the effect of the threshold level on robustness, we also analyze the genetic robustness at different threshold levels (from T1 to T9). Figure 4 shows the number of significantly robust miRNAs with FDR-controlled P-values of $<0.05,<0.01,<0.005$, and $<0.001$ at different threshold levels, where the robustness $\gamma_{i}^{m}, \mathrm{i}=1,2, \cup, 9$ for each real pre-miRNA is compared to that of 1,000 random sequences and pseudo pre-miRNA sequences at each threshold level. Increased threshold levels result in a rapid increase in the number of robust miRNA sequences. After threshold level T6, the number of miRNAs with FDR-controlled P-values of $<0.05,<0.01,<0.005$, and $<0.001$ as filters is almost identical, and is almost equal to 1,082 at T9. Similarly, we also show the number of significantly robust miRNAs with different FDR-controlled P-values at different threshold levels, where the robustness $\gamma_{i}^{m}, \mathrm{i}=1$,

Table I: Z-scores of the robustness $\gamma_{1}^{m}$ at threshold level $T_{1}$. Rates for each real pre-miRNA compared to I,000 random and four types of shuffled sequences.

\begin{tabular}{|c|c|c|c|c|c|}
\hline Species & Random & Zero-markov & Mononucleotide & First-markov & Dinucleotide \\
\hline H. sapiens & $-2.39 \pm 0.64$ & $-2.42 \pm 0.63$ & $-2.42 \pm 0.63$ & $-2.45 \pm 0.63$ & $-2.44 \pm 0.64$ \\
\hline C. elegans & $-2.50 \pm 0.92$ & $-2.48 \pm 0.89$ & $-2.47 \pm 0.89$ & $-2.52 \pm 0.90$ & $-2.49 \pm 0.89$ \\
\hline D. melanogaster & $-2.44 \pm 0.75$ & $-2.39 \pm 0.74$ & $-2.39 \pm 0.74$ & $-2.43 \pm 0.74$ & $-2.40 \pm 0.73$ \\
\hline D. rerio & $-2.39 \pm 0.59$ & $-2.40 \pm 0.58$ & $-2.40 \pm 0.57$ & $-2.44 \pm 0.57$ & $-2.4 I \pm 0.57$ \\
\hline M. musculus & $-2.23 \pm 0.79$ & $-2.25 \pm 0.77$ & $-2.26 \pm 0.77$ & $-2.29 \pm 0.77$ & $-2.27 \pm 0.76$ \\
\hline R. norvegicus & $-2.32 \pm 0.78$ & $-2.37 \pm 0.75$ & $-2.37 \pm 0.76$ & $-2.40 \pm 0.76$ & $-2.37 \pm 0.75$ \\
\hline Average & $-2.37 \pm 0.71$ & $-2.38 \pm 0.69$ & $-2.38 \pm 0.69$ & $-2.42 \pm 0.70$ & $-2.40 \pm 0.69$ \\
\hline
\end{tabular}


Table 2: Z-scores of the robustness $\gamma_{1}^{m}$ at threshold level $T_{1}$. Rates for each real pre-miRNA compared to 1,000 random and four types of shuffled pseudo pre-miRNAs.

\begin{tabular}{cccccc}
\hline Species & Random & Zero-markov & Mononucleotide & First-markov & Dinucleotide \\
\hline H. sapiens & $-2.11 \pm 0.62$ & $-2.14 \pm 0.61$ & $-2.14 \pm 0.61$ & $-2.17 \pm 0.61$ & $-2.17 \pm 0.62$ \\
C. elegans & $-2.18 \pm 0.89$ & $-2.17 \pm 0.86$ & $-2.16 \pm 0.86$ & $-2.20 \pm 0.87$ & $-2.18 \pm 0.84$ \\
D. melanogaster & $-2.14 \pm 0.74$ & $-2.11 \pm 0.71$ & $-2.07 \pm 0.80$ & $-2.15 \pm 0.72$ & $-2.15 \pm 0.72$ \\
D. rerio & $-2.07 \pm 0.61$ & $-2.11 \pm 0.56$ & $-2.13 \pm 0.67$ & $-2.13 \pm 0.56$ & $-2.11 \pm 0.55$ \\
M. musculus & $-1.96 \pm 0.77$ & $-2.00 \pm 0.74$ & $-2.00 \pm 0.75$ & $-2.04 \pm 0.75$ & $-2.02 \pm 0.73$ \\
R. norvegicus & $-2.02 \pm 0.75$ & $-2.07 \pm 0.73$ & $-2.08 \pm 0.73$ & $-2.09 \pm 0.73$ & $-2.07 \pm 0.72$ \\
Average & $-2.07 \pm 0.70$ & $-2.10 \pm 0.67$ & $-2.10 \pm 0.68$ & $-2.13 \pm 0.67$ & $-2.11 \pm 0.66$
\end{tabular}

$2, \cup, 9$ of each real pre-miRNA, is compared with that of 1,000 shuffled sequences and shuffled pseudo pre-miRNAs at each threshold level, respectively (Additional files 5 and 6). These data demonstrate the threshold levels have not affect robustness and the different types of shuffled methods are indistinguishable (Figure 4 and Additional files 5 and 6 ).

Due to the correlation between the thermodynamic stability of the minimum free energy structure of a given sequence and its genetics robustness [55], the increased genetic robustness described above may arise from the increased thermodynamic stability of miRNAs, as recently reported by Bonnet et al. [21]. If so, genetic robustness may have evolved as a correlated side effect of environmental robustness. Here, we not only examine the thermodynamic stability of miRNAs in an analogous manner

(a)

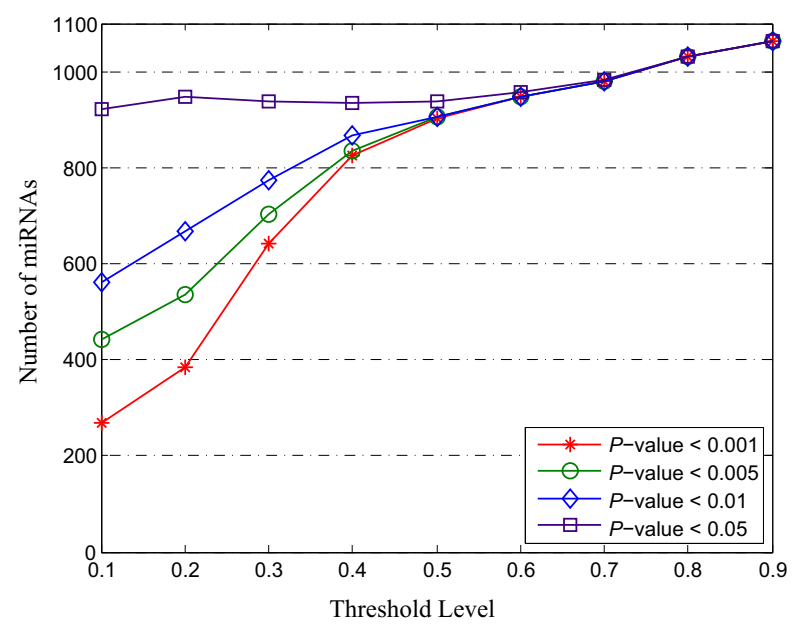

to that done by Bonnet et al. [21] but also using a background model, based on random and shuffled pseudo pre-miRNAs, that maintain the stem-loop structure as in the real pre-miRNA (see Methods). Figure 5 graphs Pvalue against $Z$-score of environmental robustness for all the 1,082 miRNAs, and the corresponding P-value distribution is showed in Figure 6. Comparison with random sequences suggests 917 out of 1,082 (84.8\%) show a high level of thermodynamic stability at FDR-controlled Pvalue of $<0.05$, similar to that previously reported [21] (Figure 7, Table 3, and Table S3, Additional file 15). As comparing with random pseudo pre-miRNAs, significant thermodynamic stability is also observed in most miRNAs (904 of 1,082, 83.5\%) at FDR-controlled P-value of < 0.05 , suggesting that it is not a byproduct of the special stem-loop structure (Figure 7, Table 4, and Table S4, Additional file 15). There are also a high proportion of miRNA sequences at each FDR-controlled P-value (Figure 7, Table

(b)

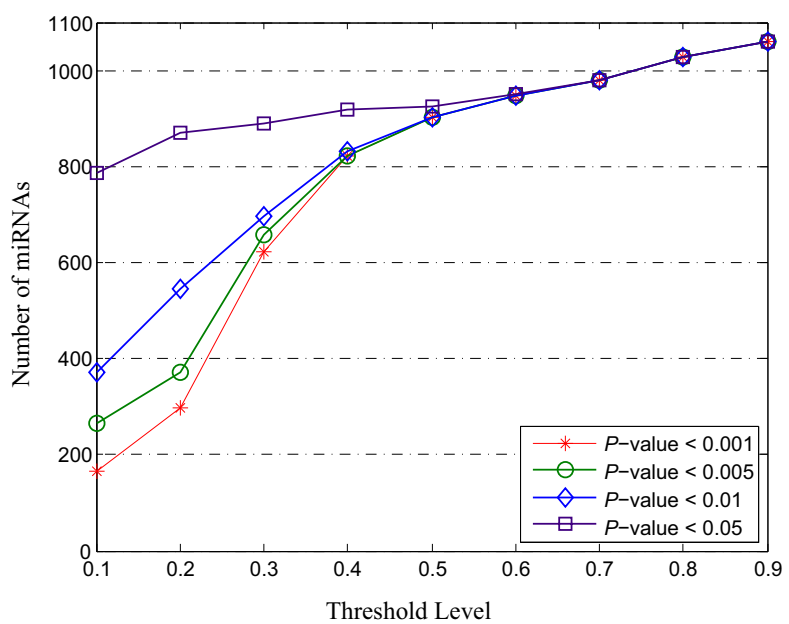

Figure 4

Number of genetically robust miRNAs with FDR-controlled $P$-values of $<0.05,0.01,0.005$, and 0.001 at different threshold levels. For each real pre-miRNA, the robustness $\gamma_{i}^{m}, i=\mathrm{I}, 2, \cup, 9$ is compared with I,000 random sequences (a) and random pseudo pre-miRNAs (b). 
(a)

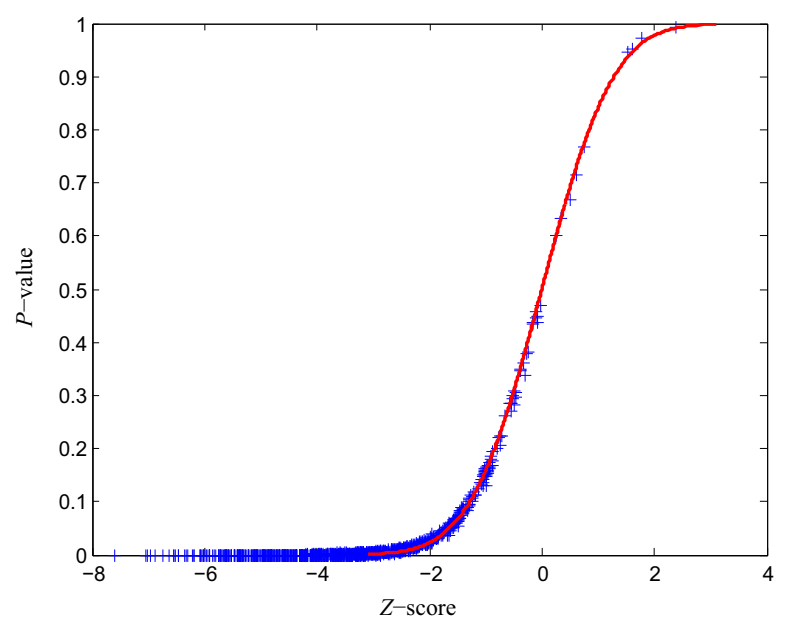

(b)

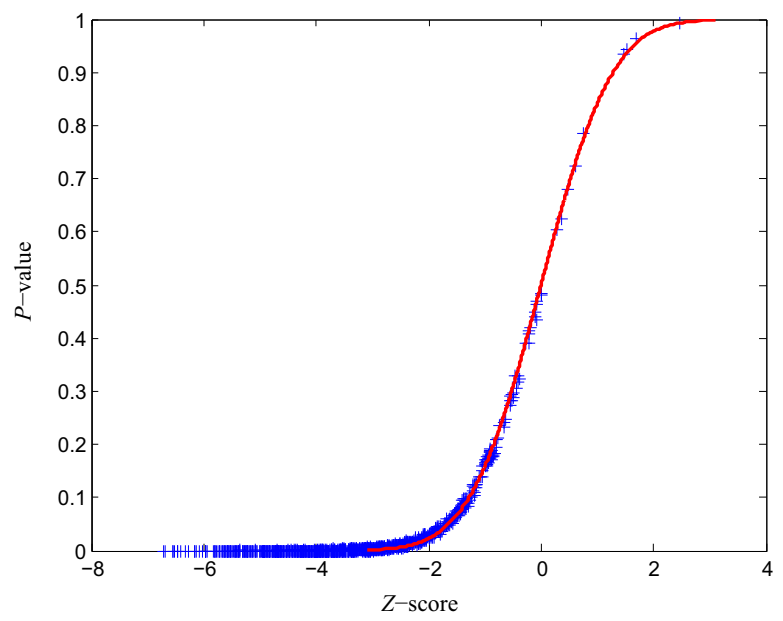

Figure 5

Correlation between Z-scores and P-values of environmental robustness for all the I,082 miRNAs. For each real pre-miRNA, the free energy is compared with that of I,000 random sequences (a) and random pseudo pre-miRNAs (b), and the $Z$-score and $P$-value are computed. The curve for a normal distribution of mean 0 and a standard deviation of $I$ is also displayed.

3, Additional files 7 and 8, and Table S3, Additional file 15), when compared with the four types of shuffling sequences. Over $90 \%$ of the 1,082 miRNA sequences show a high level of thermodynamic stability at FDR-controlled P-value of $<0.05$, based on four types of shuffled methods (Figure 7, Table 3 and Table S3, Additional file $15)$. There is also a high proportion of miRNAs $(>72 \%$ for the four types of shuffled methods) with FDR-controlled P-value $<0.005$ as filter (Figure 7, Table 3, and Table S3,

(a)

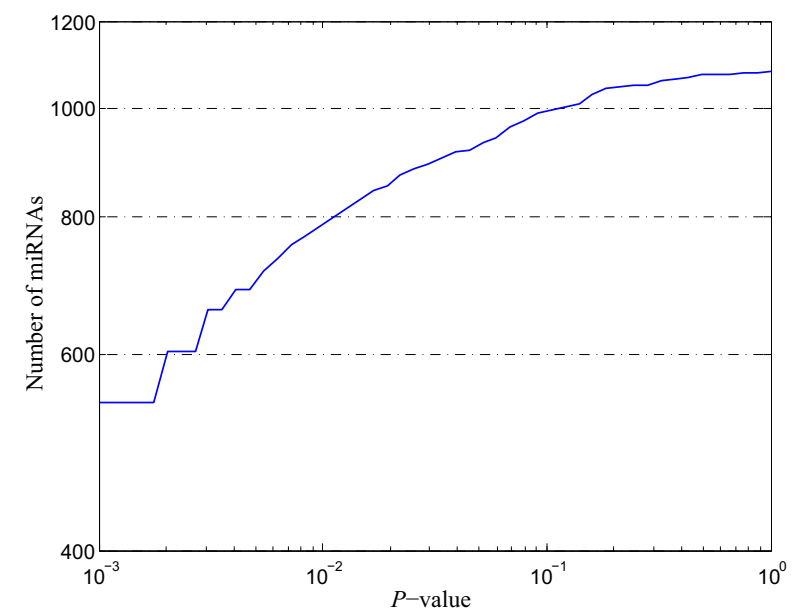

Additional file 15). The reference sets of shuffled pseudo pre-miRNAs generated for each miRNA are utilized to further confirm that the increased thermodynamic stability is not the product of the specific stem-loop structure (Figure 7, Additional files 9 and 10, Table 4, and Table S4- Additional file 15). Our data suggest that more than 1010 of the 1,082 (>93\% for the four types of shuffled methods) miRNA sequences show a high level of thermodynamic stability at FDR-controlled P-value of $<0.05$, with a high

(b)

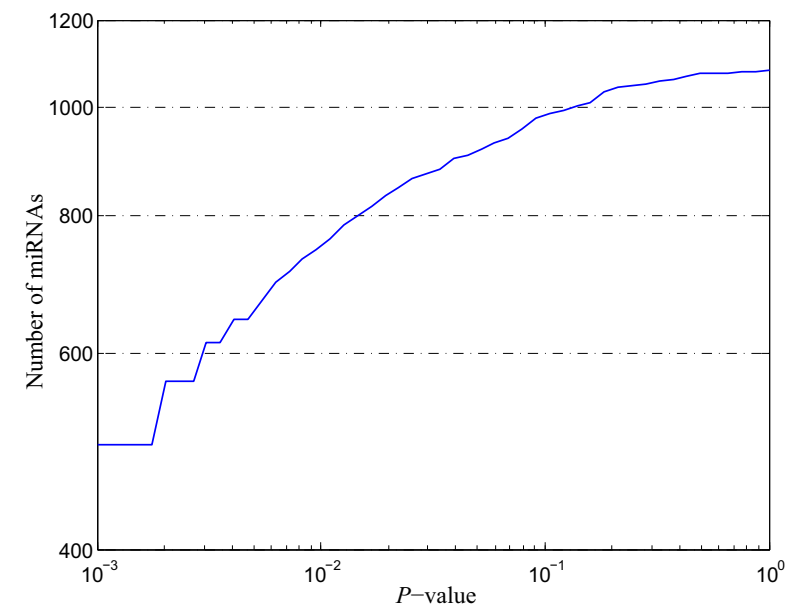

Figure 6

P-value distribution of environmental robustness for the $\mathbf{I , 0 8 2}$ miRNAs. For each real pre-miRNA, the free energy is compared with that of 1,000 random sequences (a) and random pseudo pre-miRNAs (b). 


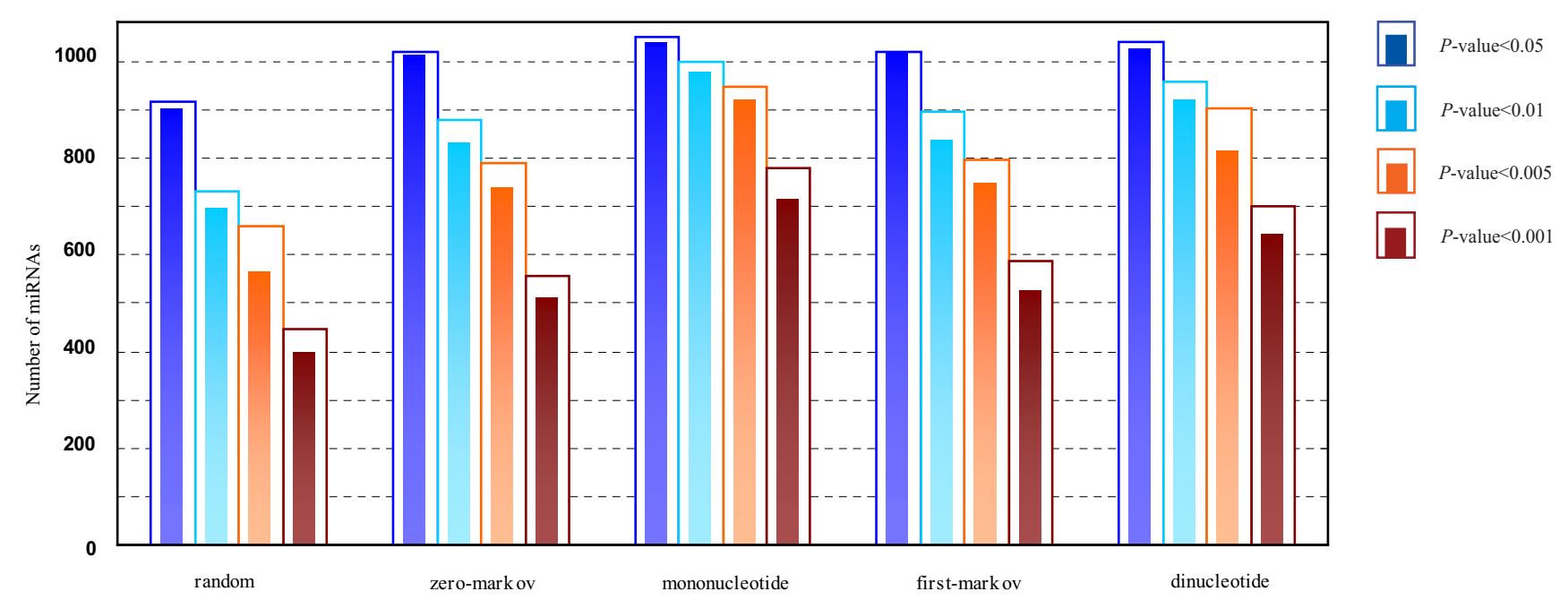

\section{Figure 7}

Number of miRNAs with significantly environmental robustness at FDR-controlled $P$-values of $<0.05,0.0 \mathrm{I}$, 0.005 , and $0.00 \mathrm{I}$. 2D histogram plots of the number of miRNAs with significantly genetic and environmental robustness. Each bar is constituted with an outer hollow sub-bar and an inner solid sub-bar, which represents the number of significantly robust real pre-miRNAs compared with random/shuffled sequences and random/shuffled pseudo pre-miRNAs, respectively.

proportion ( $>68 \%$ for the four types of shuffled methods) is also observed at FDR-controlled P-value of $<0.005$ (Figure 7, Table 4, and Table S4- Additional file 15). Additionally, examining the thermodynamic stability of miRNA genes within species provides a similar picture for each species separately (Figure 7, Additional files 7, 8, 9, 10, Table 3 - 4, and Additional file 15, Tables S3-S4).

We also investigate the correlation between genetic robustness and thermodynamic stability of miRNA. Figure 8 shows the scatter plots of Z-scores of genetic and environmental robustness for all the 1,082 miRNAs. Although a strong correlation is not observed (Pearson's correlation coefficient is $0.43,0.40$ for random sequences and random pseudo pre-miRNAs, respectively), most of the significantly genetic robust miRNA genes exhibit significant thermodynamic stability at the same time. Of the 1,082 miRNA genes investigated, 789 (72.9\%) and 662
(61.2\%) miRNAs are showed significantly genetic and environmental robustness with FDR-controlled P-value of $<0.05$ (Figure 9, Table S5 and S6 Additional file 15), compared with random sequences and random pseudo premiRNAs, respectively. These also can be seen from the $3 \mathrm{D}$ histogram plots of Z-scores of genetic and environmental robustness (Figure 10). Furthermore, the correlation between the genetic and environmental robustness is not a byproduct of a base composition bias (Additional files 11, 12, 13, 14 and Tables S5-S6 Additional file 15). Our results demonstrate that the stem-loop structures of miRNAs buffer against genetic variations, at the same time is also insensitive to environmental perturbations, indicating that the increase in genetic robustness may evolve as a correlated side effect of the evolution for environmental robustness.

Table 3: Z-scores of free energy. Comparison between each real pre-miRNA and I,000 random and four types of shuffled sequences.

\begin{tabular}{|c|c|c|c|c|c|}
\hline Species & Random & Zero-markov & Mononucleotide & First-markov & Dinucleotide \\
\hline H. sapiens & $-3.96 \pm 1.27$ & $-4.31 \pm 1.40$ & $-4.36 \pm 1.40$ & $-6.05 \pm 1.85$ & $-5.63 \pm 1.87$ \\
\hline C. elegans & $-2.51 \pm 1.52$ & $-3.34 \pm 1.45$ & $-3.37 \pm 1.46$ & $-4.85 \pm 2.18$ & $-4.87 \pm 2.09$ \\
\hline D. melanogaster & $-2.30 \pm 1.05$ & $-3.53 \pm 1.08$ & $-3.53 \pm 1.08$ & $-4.83 \pm|.5|$ & $-4.85 \pm 1.56$ \\
\hline D. rerio & $-2.87 \pm 1.33$ & $-3.53 \pm 1.19$ & $-3.64 \pm 1.23$ & $-4.91 \pm 1.69$ & $-4.67 \pm 1.64$ \\
\hline M. musculus & $-3.63 \pm 1.25$ & $-3.89 \pm 1.40$ & $-3.87 \pm 1.34$ & $-5.41 \pm 1.83$ & $-4.95 \pm 1.84$ \\
\hline R. norvegicus & $-3.68 \pm 1.33$ & $-3.60 \pm 1.30$ & $-3.68 \pm 1.33$ & $-5.25 \pm 1.80$ & $-4.80 \pm 1.86$ \\
\hline Average & $-3.26 \pm 1.42$ & $-3.75 \pm 1.35$ & $-3.81 \pm 1.35$ & $-5.28 \pm 1.86$ & $-4.98 \pm 1.83$ \\
\hline
\end{tabular}


Table 4: Z-scores of free energy. Comparison between each real pre-miRNA and I,000 random and four types of shuffled pseudo premiRNAs

\begin{tabular}{cccccc}
\hline Species & Random & Zero-markov & Mononucleotide & First-markov & Dinucleotide \\
\hline H. sapiens & $-3.81 \pm 1.22$ & $-4.16 \pm 1.38$ & $-4.22 \pm 1.36$ & $-5.73 \pm 1.78$ & $-5.27 \pm 1.79$ \\
C. elegans & $-2.39 \pm 1.49$ & $-3.22 \pm 1.42$ & $-3.25 \pm 1.42$ & $-4.57 \pm 2.05$ & $-4.59 \pm 2.01$ \\
D. melanogaster & $-2.20 \pm 1.03$ & $-3.42 \pm 1.06$ & $-3.43 \pm 1.04$ & $-4.57 \pm 1.46$ & $-4.61 \pm 1.52$ \\
D. rerio & $-2.74 \pm 1.29$ & $-3.40 \pm 1.16$ & $-3.51 \pm 1.19$ & $-4.61 \pm 1.62$ & $-4.37 \pm 1.58$ \\
M. musculus & $-3.48 \pm 1.20$ & $-3.75 \pm 1.37$ & $-3.74 \pm 1.33$ & $-5.12 \pm 1.76$ & $-4.62 \pm 1.78$ \\
R. norvegicus & $-3.52 \pm 1.25$ & $-3.44 \pm 1.26$ & $-3.51 \pm 1.29$ & $-4.96 \pm 1.74$ & $-4.46 \pm 1.77$ \\
Average & $-3.12 \pm 1.37$ & $-3.62 \pm 1.32$ & $-3.68 \pm 1.32$ & $-4.98 \pm 1.78$ & $-4.66 \pm 1.76$ \\
\hline
\end{tabular}

\section{Discussion}

There has been a growing interest of evolutionary biologists in the origins, mechanisms and consequences of robustness [2], which is a fundamental and ubiquitously observed property of biological systems [8]. A greater understanding of the evolution of robustness is needed to clarify whether genetic robustness evolved as a direct consequence of natural selection (adaptive robustness), as a byproduct of stabilizing selection acting on fitness-related traits (intrinsic robustness), or as a correlated response to environmental robustness (congruent robustness) [2]. Recent studies have demonstrated that the stem-loop structures of miRNA are tolerant to some structural changes and show thermodynamic stability. We therefore hypothesize that the genetic robustness of miRNAs may evolve as a correlated side effect of the evolution for environmental robustness in the current study. Using the method proposed in our previous study [54], we examine the robustness of 1,082 miRNA genes covering six species, and show that the stem-loop structures of miRNA precur-

(a)

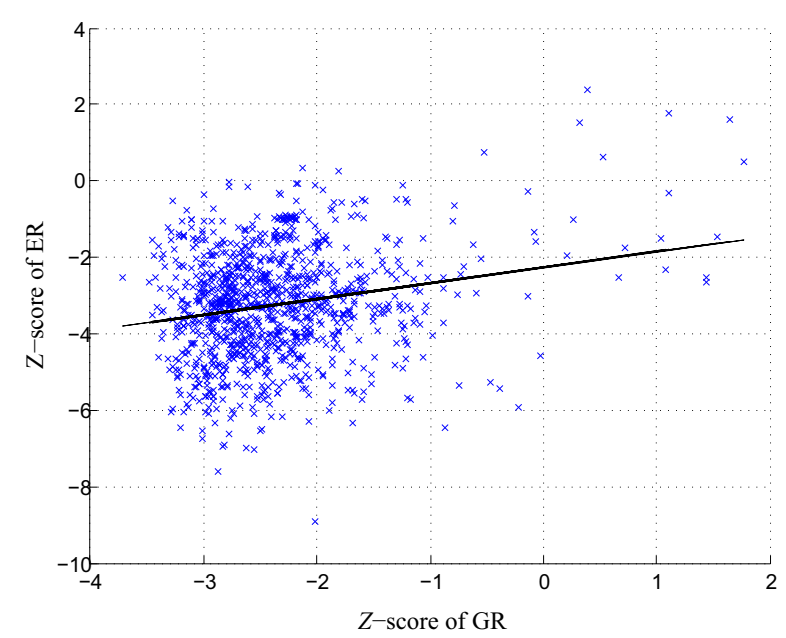

sors exhibit a significantly higher level of genetic robustness at different FDR-controlled P-values. Additionally, we generate a reference background of phenotypes similar to real pre-miRNAs (pseudo pre-miRNAs), and demonstrate that this excess robustness of miRNA genes goes beyond the intrinsic robustness of the stem-loop structure. The effect of base composition bias on robustness is ruled out by careful design of corresponding shuffling reference backgrounds. Examination of the environmental robustness of real pre-miRNAs also demonstrates that the phenotype of miRNA buffers against genetic perturbations, and at the same time is insensitive to environmental perturbations. These results suggest that increased genetic robustness may evolve as a correlated side effect of the evolution for environmental robustness.

Pang et al. [56] argue that even slight changes in miRNA sequences can fundamentally alter their function, when they interpret the conservation of miRNAs and snoRNAs. Our data suggest this interpretation might be premature.

(b)

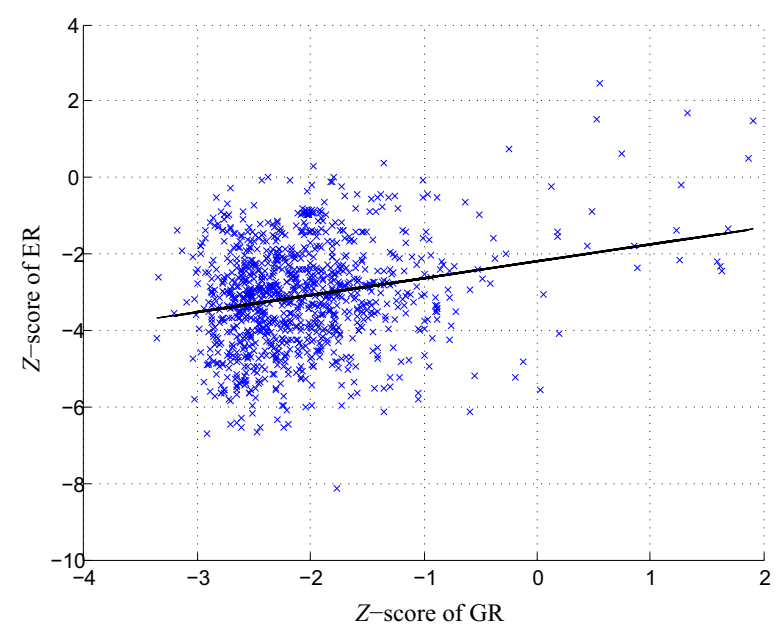

Figure 8

Correlation between genetic and environmental robustness. Scatter plots of Z-scores of genetic robustness and environmental robustness. The $Z$-scores are obtained by comparing the robustness of real pre-miRNAs with that of I,000 random sequences (a) and random pseudo pre-miRNAs (b). 


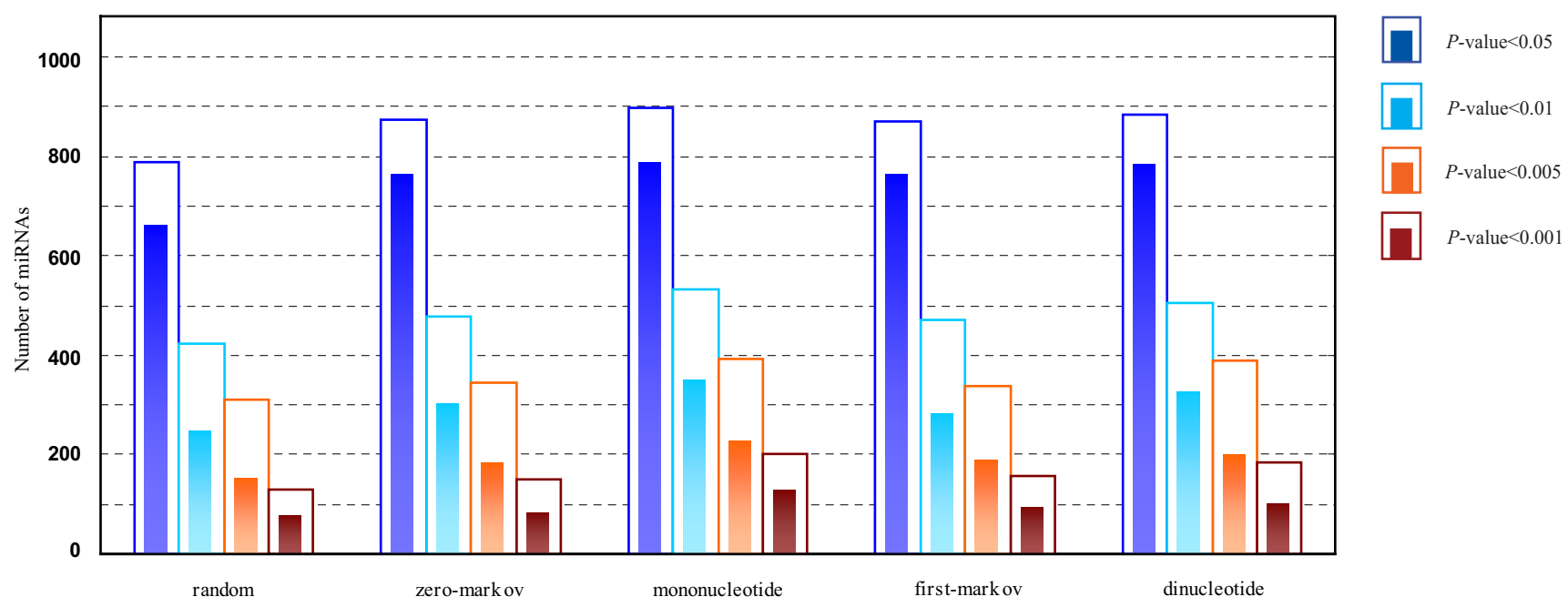

\section{Figure 9}

Number of miRNAs with significantly genetic and environmental robustness at FDR-controlled $P$-values of $<$ $0.05,0.01,0.005$, and $0.00 \mathrm{I}$. 2D histogram plots of the number of miRNAs with significantly genetic and environmental robustness. Each bar is constituted with an outer hollow sub-bar and an inner solid sub-bar, which represents the number of significantly robust real pre-miRNAs compared with random/shuffled sequences and random/shuffled pseudo pre-miRNAs, respectively.

Experimental studies also have demonstrated that stemloop structures of miRNAs are, to a certain degree, tolerant to some structural changes, as exemplified by studies in which no effects are noted resulting from changes in the loop sequence [30-33] and further suggesting that the

(a)

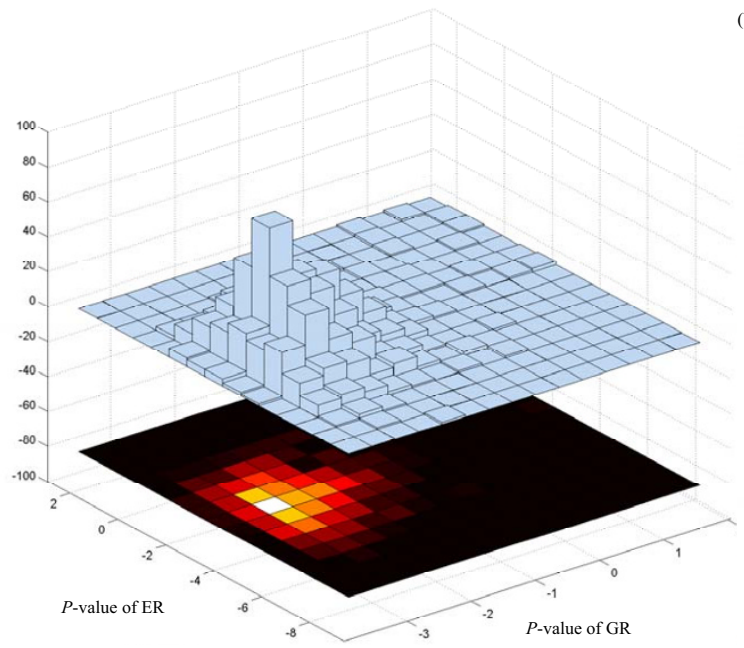

sequence/structure requirements for miRNA processing are quite lax, in agreement with our results.

A recent study, published during the course of our work, reports that the stem-loop structures of miRNA precursors show excess robustness with respect to mutational pertur-

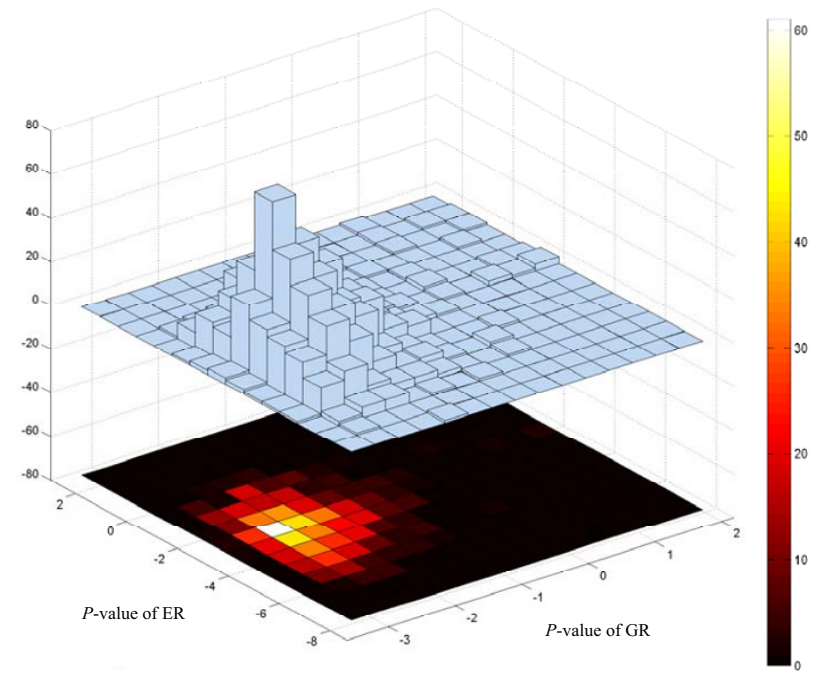

\section{Figure 10}

Distribution of Z-score of genetic and environmental robustness. 3D histogram plots of Z-scores of genetic and environmental robustness. The Z-scores are obtained by comparing the robustness of real pre-miRNAs with that of 1,000 random sequences (a) and random pseudo pre-miRNAs (b). 
bation, compared with random RNA sequences with similar stem-loop structures [57], suggesting that this excess robustness of miRNAs goes beyond the intrinsic robustness of the stem-loop hairpin structure. The study further demonstrates that this excess robustness is not the byproduct of a base composition bias. These results are in much agreement with our findings, although the quantitative measures of genetic robustness defined in these two studies are quite different. The authors then reexamine the thermodynamic stability of miRNA utilizing the method used by Bonnet et al. [21], with a background model based on inversely folded sequences rather than the shuffled sequences, and find that most of the statistical effect vanished, suggesting that the excess robustness of miRNA stem-loops is the result of direct evolutionary pressure toward increased robustness. The different conclusions (adaptive robustness vs. congruent robustness) may have resulted from differences in the reference backgrounds employed. The reference backgrounds in our study are made up of random and shuffled pseudo pre-miRNA sequences with preserved phenotypes that are similar to real pre-miRNAs (see Methods). Their reference backgrounds, on the other hand, are produced by inverse folding.

On the other hand, Hermission and Wagner classify robustness as adaptive and intrinsic [58]. The adaptive robustness in their definition encompass both adaptive and congruent scenarios within the classification system established by de Visser et al. [2]. They consider robustness to be adaptive if the buffering of that trait with respect to some source of variation has the target of natural selection. In other words, robust character states are selected because of their reduced variability. According to this view, our study and the study of Borenstein and Ruppin [57] come to the same conclusion of adaptive robustness, which is a property that evolves for its own sake. The difference is that the natural forces, assumed to be responsible for its evolution, function as a kind of stabilizing selection acting directly on a character, or on some highly correlated pleiotropic trait [58].

Our study will facilitate the in silico identification of novel miRNAs. Computational identification of miRNAs is based largely on the phylogenetic conservation and the structural characteristics of miRNA precursors [59-61]. A recent study integrating bioinformatics predictions with microarray analysis and sequence-directed cloning, that does not include inter-species conservation, identifies hundreds of new human miRNAs, many of which are poorly conserved beyond primates [62]. The significantly greater robustness of the miRNA stem-loop structures that buffers environmental and genetic perturbations may also facilitate improved miRNA identification on a single genome and can serve as a complementary method to fil- ter out random pseudo pre-miRNA sequences. Our findings may also be utilized for the improvement of in vitro selection or SELEX (Systematic Evolution of Ligands by Exponential enrichment), an experimental method for selecting functional RNAs from a large pool $\left(10^{15}\right)$ of random sequences $[63,64]$. The use of designed sequences with thermodynamic stability and genetic robustness, in lieu of random sequences, may increase the probability of identifying novel functional RNAs.

The in silico approach utilized in the current study is a simple yet powerful, biologically well-grounded model for studying the evolution of genetic robustness. The importance of this computational approach is emphasized by the fact that the large numbers of perturbations (more than $2 \times 10^{9}$ point mutants are generated and folded) analyzed here are not easily studied in laboratory experiments. Although this in silico study can not completely rule out all alternative hypotheses, the carefully designed reference background model and the statistical analysis of the results strongly support the hypothesis of congruent evolution of robustness. A greater understanding of the evolution of robustness will require quantitative knowledge of the forces producing robustness, such as the distribution of fitness effects of mutations [12]. Further researches are needed to fully elucidate the mechanisms of the evolution of robustness.

\section{Conclusion}

The current study investigate the evolutionary origin of genetic robustness - a fundamental evolutionary molecular biology problem which has not been fully elucidated. We have shown that miRNA stem-loop structures exhibit a significantly higher level of genetic robustness at different FDR-controlled P-values, which goes beyond the intrinsic robustness of the stem-loop structure and is not a byproduct of a base composition bias. Furthermore, we have demonstrated that the phenotypes of miRNAs buffer against genetic perturbations, and at the same time are also insensitive to environmental perturbations. Our findings suggest that the increased robustness of miRNA stemloops may evolve as a correlated side effect of evolution for environmental robustness.

\section{Methods \\ Real pre-miRNAs, reference sets and RNA folding}

1,082 real pre-miRNA sequences included in the analysis are selected from MicroRNA Registry release 7.1, which all have been experimentally verified to avoid a possible bias introduced by consideration of predicted miRNA precursors (Table 5) $[65,66]$. The available sequences covered six species: $H$. sapiens, D. melanogaster, D. rerio, C. elegans, $M$. musculus, and R. norvegicus. 
Table 5: Robustness analysis of miRNA within each species

\begin{tabular}{cccccc}
\hline Species & $N_{s}$ & Length & \%GC & $\begin{array}{c}\text { No. of genetically } \\
\text { robust miRNAs (\%) }\end{array}$ & $\begin{array}{c}\text { No. of } \\
\text { environmentally } \\
\text { robust miRNAs (\%) }\end{array}$ \\
\hline H. sapiens & 242 & $85.48 \pm 14.49$ & $47.67 \pm 7.88$ & $177(73.1)$ & $233(96.3)$ \\
C. elegans & 112 & $98.29 \pm 6.42$ & $44.61 \pm 6.89$ & $93(83.0)$ & $74(66.1)$ \\
D. melanogaster & 75 & $87.67 \pm 12.74$ & $41.60 \pm 5.39$ & $54(72.0)$ & $48(64.0)$ \\
D. rerio & 350 & $94.36 \pm 18.49$ & $45.11 \pm 6.51$ & $254(72.6)$ & $254(72.6)$ \\
M. musculus & 191 & $80.26 \pm 12.48$ & $48.19 \pm 8.26$ & $130(68.1)$ & $177(92.7)$ \\
R. norvegicus & 112 & $90.96 \pm 9.14$ & $50.34 \pm 7.96$ & $77(68.8)$ & $105(93.8)$
\end{tabular}

$N_{s}$, total number of experimentally verified miRNA sequences; Length, distribution of miRNA sequence length; \%GC, distribution of GC content of miRNA sequences; No. of genetically (environmentally) robust miRNAs (\%), the number of significantly genetically (environmentally) robust miRNAs (\%) at FDR-controlled $P$-value of $<0.05$, comparing with random pseudo pre-miRNAs.

In addition to the real pre-miRNAs specified in Table 5, a reference set consisting of 1,000 random RNA sequences for each real pre-miRNA is generated, and an additional reference set consisting of 1,000 hairpin sequences with similar stem-loops as real pre-miRNAs (pseudo pre-miRNAs) using a similar idea of the RNAinverse program in Vienna RNA package [53] is also generated to investigate whether the increased robustness stemmed intrinsically from the miRNA stem-loop structures. To rule out the effect of base composition bias on robustness, four types of reference sets for each real pre-miRNA are made using four different sequence shuffling methods: mononucleotide shuffling, dinucleotide shuffling and shuffling based on a zero and first order Markov model. Each type of reference set is also consisted of 1,000 shuffled sequences that preserved the exact or nearly exact mononucleotide and dinucleotide frequencies as real premiRNA. Additionally, four types of shuffling reference sets which consisted of 1,000 shuffled pseudo pre-miRNAs that preserved not only the stem-loop structure but also the exact or nearly exact mononucleotide and dinucleotide frequencies as real pre-miRNA are generated for each miRNA. These sequence shuffling methods first generate the corresponding shuffling sequences preserving exact or nearly exact mononucleotide and dinucleotide frequencies with the methods previously used in the studies of the thermodynamic stability of RNA secondary structures [21$23,26]$, and then search for the shuffling sequences with similar stem-loop as real pre-miRNAs using a similar idea of the RNAinverse program [53].

The RNAfold in Vienna RNA package (version 1.6) [53] is utilized with default parameter values $\left(T=37^{\circ} \mathrm{C}\right)$ to predict the secondary structures, based on Zuker's minimum free energy algorithm [52]. The current study only utilized optimal folding results.

\section{Robustness evaluation}

Experimental researches have demonstrated that the secondary structures of miRNAs are tolerant to some struc- tural changes [30-33]. To reflect this flexibility in sequence/structure requirements, at a given threshold, $T_{j}$, we define the robustness [54], $\gamma_{j}$, as follows:

$$
\gamma_{j}=\left\langle N_{j}(d)\right\rangle, j=1,2, \ldots, 9
$$

where $d$ is the structure distance between the secondary structure of the WT sequence and the secondary structure of the mutant, and $N_{j}(d)$ is number of mutants with structure distance lesser than or equal to the threshold $T_{j} . \gamma_{j}$ is the average of $N_{j}(d)$ over all $3 \times L$ one-mutant neighbors. The maximum value of the structural distance between the secondary structure of the WT sequence and the secondary structure of the mutants is used as a baseline value to evaluate the threshold level [54]. The threshold $T_{j}, j=1$, $2, \ldots, 9$ is set to $10 \%, 20 \%, \ldots, 90 \%$ of the maximum value of the metric, respectively. The larger value of the robustness $\gamma_{j}$ at threshold $T_{j}$ indicates a relatively higher level of robustness.

We compare the secondary structure between WT and its mutant using a variety of distance measures for secondary structures [53,67-69], including topological indices [70], tree-edit and string-eidt distance $[53,71]$, and base-pair distance [55]. While the data in this paper are obtained by base-pair distance, the qualitative results are obtained independent of the distance measure used.

\section{Statistical significance analysis}

At each threshold $T_{j}$, we evaluate the robustness $\gamma_{j}^{m}$ of the real pre-miRNA and $\Upsilon_{j}=\left\{\gamma_{j}^{r_{i}}, i=1,2, \ldots, N\right\}$ of the corresponding 1,000 random sequences in reference set $X(N$ is the number of the sequences in the reference set, $|X|)$, then compare $\gamma_{j}^{m}$ with $\Upsilon_{j}$. Z $Z$-score and $P$-value are employed here to determine whether the phenotype of a real pre-miRNA sequence shows significantly robust from that of reference sequences, which have been widely 
applied in statistical significance analysis [21-24,26,72]. The $Z$-score is the number of standard deviations by which $\gamma_{j}^{m}$ of a real pre-miRNA sequence differs from the mean $\gamma_{j}^{r_{i}}, i=1,2, \ldots, N$ of the random reference sequences set $X$ and is defined as:

$$
Z\left(\gamma_{j}^{m}\right)=\frac{<\Upsilon_{j}>-\gamma_{j}}{\sigma\left(\Upsilon_{j}\right)}, \quad j=1,2, \ldots, 9
$$

where $<\cdot>$ and $\sigma(\cdot)$ denote the mean and the standard deviation of the $\Upsilon_{j}$. The $P$-value of $\gamma_{j}^{m}$ of a real pre-miRNA is the fraction of sequences in $X$ having robustness larger than the real pre-miRNA sequence, that is, the area under the distribution function to the right of the $\gamma_{j}^{m}$, and is defined as:

$$
P\left(\gamma_{j}^{m}\right)=\frac{M}{N+1}, \quad j=1,2, \ldots, 9
$$

where $M$ is the number of sequences in $X$ with larger robustness than $\gamma_{j}^{m}$ of the real pre-miRNA sequence.

The statistical significance analysis of environmental robustness is similar to that done for genetic robustness, in which the robustness $\gamma_{j}$ at a threshold $T_{j}$ is simply replaced by the free energy of the sequences. The thermodynamic stability of pre-miRNAs is examined as previously reported [21], but also used a background model based on the random and shuffled pseudo pre-miRNAs.

\section{False discovery rate}

Because the above statistical significance analysis involves the simultaneous testing of thousands of hypotheses, multiple hypotheses testing is important to control the overall Type I error rate. We will use direct control of the false discoveries using the commonly applied FDR criterion. The FDR, based on the outcomes of $m$ statistical tests (Table 6), is defined as the expected proportion of false positives among the rejected hypotheses, i.e.

$$
F D R=E\left(\frac{V}{R} \mid R>0\right) \cdot \operatorname{Pr}(R>0)
$$

where $V$ is the number of false positives and $R$ is the number of rejected hypotheses. Therefore, an FDR cut-off has a meaningful interpretation.

To compute the FDR, we apply the Benjamini Hochberg procedure [73]. Considering testing $H_{1}, H_{2}, \cup, H_{m}$ based on the corresponding $P$-values $P_{(1)}, P_{(2)}, \cup, P_{(m)}$. Let $P_{(1)} \leq$
Table 6: Possible outcomes of the $m$ statistical tests.

\begin{tabular}{lccc}
\hline & $\begin{array}{c}\text { Called not } \\
\text { significant }\end{array}$ & Called significant & Total \\
\hline Null true & $U$ & $V$ & $m_{0}$ \\
Alternative true & $T$ & $S$ & $m-m_{0}$ \\
Total & $M-R$ & $R$ & $m$ \\
\hline
\end{tabular}

$N_{s}$, total number of experimentally verified miRNA sequences; Length, distribution of miRNA sequence length; \%GC, distribution of GC content of miRNA sequences; No. of genetically (environmentally) robust miRNAs (\%), the number of significantly genetically (environmentally) robust miRNAs (\%) at FDR-controlled $P$-value of < 0.05 , comparing with random pseudo pre-miRNAs.

$P_{(2)} \leq \cup \leq P_{(m)}$ be the ordered $P$-values, and denote by $H_{(i)}$ the null hypothesis corresponding to $P_{(i)}$. Define

$$
k=\max \left\{i: P_{(i)} \leq \frac{i}{m} q^{*}\right\}
$$

and reject all $H_{(i)}, i=1,2, \cup, k$. If no such $i$ exists, reject no hypothesis.

\section{Competing interests}

The author(s) declare that they have no competing interests.

\section{Authors' contributions}

WS wrote the programs, analyzed the results and drafted the manuscript. $\mathrm{XB}, \mathrm{MN}$ and $\mathrm{ZZ}$ helped in analysis and discussion, gave useful comments. SW and $\mathrm{XB}$ guided the project. All authors read and approved the final manuscript.

\section{Additional material}

\section{Additional File 1}

Correlation between $Z$-scores and P-values of genetic robustness for all the 1,082 miRNAs and all four types of shuffled mothods at threshold level $\mathrm{T}_{1}$. For each real pre-miRNA, the robustness $\gamma_{1}^{m}$ is compared with that of 1,000 zero-markov shuffling sequences (a), monoshuffling sequences (b), first-markov shuffling sequences (c) and dishuffling sequences (d), and the Z-score and $\mathrm{P}$-value are computed. The curve for a normal distribution of mean 0 and a standard deviation of 1 is also displayed. Click here for file

[http://www.biomedcentral.com/content/supplementary/1471 2148-7-223-S1.pdf] 


\section{Additional File 2 \\ P-value distribution of genetic robustness for the 1,082 miRNAs at thresh- old level $\mathrm{T}_{1}$. For each real pre-miRNA, the robustness $\gamma_{1}^{m}$ is compared with that of 1,000 zero-markov shuffling sequences (a), monoshuffling sequences (b), first-markov shuffling sequences (c) and dishuffling sequences (d), and the Z-score and P-value are computed. Click here for file \\ [http://www.biomedcentral.com/content/supplementary/1471- 2148-7-223-S2.pdf]}

\section{Additional File 3}

Correlation between Z-scores and P-values of genetic robustness for all the 1,082 miRNAs and all four types of shuffled mothods at threshold level $\mathrm{T}_{1}$. For each real pre-miRNA, the robustness $\gamma_{1}^{m}$ is compared with that of 1,000 zero-markov shuffling pseudo pre-mikNAs (a), monoshuffling pseudo pre-miRNAs (b), first-markov shuffling pseudo pre-miRNAs (c) and dishuffling pseudo pre-miRNAs (d), and the Z-score and P-value are computed. The curve for a normal distribution of mean 0 and a standard deviation of 1 is also displayed.

Click here for file

[http://www.biomedcentral.com/content/supplementary/14712148-7-223-S3.pdf]

\section{Additional File 4}

P-value distribution of genetic robustness for the 1,082 miRNAs at threshold level $\mathrm{T}_{1}$. For each real pre-miRNA, the robustness $\gamma_{1}^{m}$ is compared with that of 1,000 zero-markov shuffling pseudo pre-miRNAs (a), monoshuffling pseudo pre-miRNAs (b), first-markov shuffling pseudo premiRNAs (c) and dishuffling pseudo pre-miRNAs (d), and the Z-score and P-value are computed.

Click here for file

[http://www.biomedcentral.com/content/supplementary/1471-

2148-7-223-S4.pdf]

\section{Additional File 5}

Number of miRNAs with FDR-controlled P-values of $<0.05,0.01$, 0.005 , and $0.0^{n 1}$ it different threshold levels. For each real pre-miRNA, the robustness $\gamma_{i}^{m}, \mathrm{i}=1,2, U, 9$ is compared with that of 1,000 shuffling RNA sequences based on zero-markov model (a), monoshuffling RNA sequences (b), shuffling RNA sequences based on first-markov model (c), dishuffling RNA sequences $(d)$.

Click here for file

[http://www.biomedcentral.com/content/supplementary/14712148-7-223-S5.pdf]

\section{Additional File 6}

Number of miRNAs with FDR-controlled P-values of $<0.05,0.01$, 0.005 , and 0.0n1 it different threshold levels. For each real pre-miRNA, the robustness $\gamma_{i}^{m}, \mathrm{i}=1,2, U, 9$ is compared with that of 1,000 zeromarkov shuffling pseudo pre-miRNAs (a), monoshuffling pseudo premiRNAs (b), first-markov shuffling pseudo pre-miRNAs (c) and dishuffling pseudo pre-miRNAs (d).

Click here for file

[http://www.biomedcentral.com/content/supplementary/14712148-7-223-S6.pdf]

\section{Additional File 7}

Correlation between Z-scores and P-values of environmental robustness for all the 1,082 miRNAs. For each real pre-miRNA, the free energy is compared with that of 1,000 zero-markov shuffling sequences (a), monoshuffling sequences (b), first-markov shuffling sequences (c) and dishuffling sequences $(d)$, and the Z-score and P-value are computed. The curve for a normal distribution of mean 0 and a standard deviation of 1 is also displayed.

Click here for file

[http://www.biomedcentral.com/content/supplementary/14712148-7-223-S7.pdf]

\section{Additional File 8}

P-value distributions of environmental robustness for the 1,082 miRNAs. For each real pre-miRNA, the free energy is compared with that of 1,000 zero-markov shuffling sequences (a), monoshuffling sequences (b), firstmarkov shuffling sequences (c) and dishuffling sequences (d).

Click here for file

[http://www.biomedcentral.com/content/supplementary/14712148-7-223-S8.pdf]

\section{Additional File 9}

Correlation between Z-scores and P-values of environmental robustness for all the 1,082 miRNAs. For each real pre-miRNA, the free energy is compared with that of 1,000 zero-markov shuffling pseudo pre-miRNAs (a), monoshuffling pseudo pre-miRNAs (b), first-markov shuffling pseudo pre-miRNAs (c) and dishuffling pseudo pre-miRNAs (d). The curve for a normal distribution of mean 0 and a standard deviation of 1 is also displayed.

Click here for file

[http://www.biomedcentral.com/content/supplementary/1471-

2148-7-223-S9.pdf]

\section{Additional File 10}

P-value distributions of environmental robustness for the 1,082 miRNAs. For each real pre-miRNA, the free energy is compared with that of 1,000 zero-markov shuffling pseudo pre-miRNAs (a), monoshuffling pseudo pre-miRNAs (b), first-markov shuffling pseudo pre-miRNAs (c) and dishuffling pseudo pre-miRNAs (d).

Click here for file

[http://www.biomedcentral.com/content/supplementary/14712148-7-223-S10.pdf]

\section{Additional File 11}

Correlation between genetic and environmental robustness. Scatter plots of Z-scores of genetic robustness and environmental robustness. The Zscores were obtained by comparing the robustness of real pre-miRNAs with that of 1,000 zero-markov shuffling sequences (a), monoshuffling sequences (b), first-markov shuffling sequences (c) and dishuffling sequences $(d)$.

Click here for file

[http://www.biomedcentral.com/content/supplementary/14712148-7-223-S11.pdf] 


\section{Additional File 12 \\ Correlation between genetic and environmental robustness. Scatter plots of Z-scores of genetic robustness and environmental robustness. The Z- scores were obtained by comparing the robustness of real pre-miRNAs with that of 1,000 zero-markov shuffling pseudo pre-miRNAs (a), monoshuf- fling pseudo pre-miRNAs (b), first-markov shuffling pseudo pre-miRNAs (c) and dishuffling pseudo pre-miRNAs (d). \\ Click here for file \\ [http://www.biomedcentral.com/content/supplementary/1471- 2148-7-223-S12.pdf]}

\section{Additional File 13}

Distribution of Z-score of genetic and environmental robustness. 3D histogram plots of Z-scores of genetic robustness and environmental robustness. The Z-scores were obtained by comparing the robustness of real premiRNAs with that of 1,000 zero-markov shuffling sequences (a), monoshuffling sequences (b), first-markov shuffling sequences (c) and dishuffling sequences $(d)$.

Click here for file

[http://www.biomedcentral.com/content/supplementary/14712148-7-223-S13.pdf]

\section{Additional File 14}

Distribution of Z-score of genetic and environmental robustness. 3D histogram plots of Z-scores of genetic robustness and environmental robustness. The Z-scores were obtained by comparing the robustness of real premiRNAs with that of 1,000 zero-markov shuffling pseudo pre-miRNAs (a), monoshuffling pseudo pre-miRNAs (b), first-markov shuffling pseudo pre-miRNAs (c) and dishuffling pseudo pre-miRNAs (d).

Click here for file

[http://www.biomedcentral.com/content/supplementary/1471-

2148-7-223-S14.pdf]

\section{Additional File 15}

supplementary tables included in this study.

Click here for file

[http://www.biomedcentral.com/content/supplementary/1471-

2148-7-223-S15.pdf]

\section{Acknowledgements}

The authors would like to thank the reviewers and the editors of the paper for their constructive comments which contributed to an improved presentation. The authors would also like to thank the Super Biomed Computation Center at Beijing Institute of Health Administration and Medicine Information for providing computing resources. This work is supported by a grant from the National High Technology Research and Development Program of China (No. 2007AA02Z3 II) and a grant from the National Nature Science Foundation of China (No. 30700 I39).

\section{References}

I. Wagner GP, Booth G, Bagheri-Chaichian H: A population genetic theory of canalization. Evolution 1997, 5 I:329-347.

2. de Visser JA, Hermisson J, Wagner GP, Ancel ML, Bagheri-Chaichian $\mathrm{H}$, Blanchard JL, Chao L, Cheverud JM, Elena SF, Fontana W, Gibson G, Hansen TF, Krakauer D, Lewontin RC, Ofria C, Rice SH, von Dassow G, Wagner A, Whitlock MC: Perspective: Evolution and detection of genetic robustness. Evolution Int J Org Evolution 2003, 57(9): 1959-1972.

3. Fisher RA: The possible modifications of the response of the wild type to recurrent mutations. Amer Nat 1928, 62:1 I5-1 I6.
4. Fisher RA: Two further notes on the origin of dominance. Amer Nat 1928, 62:57I-574.

5. Fisher RA: The evolution of dominance. Biological reviews 1931 , 6:345-368.

6. Waddington $\mathrm{CH}$ : The genetic assimilation of an acquired charcter. Evolution 1953, 7:1 I8-126.

7. Waddington $\mathrm{CH}$ : The strategy of the genes New York, MacMillan; 1957.

8. Kitano H: Biological robustness. Nat Rev Genet 2004, 5:826-837.

9. Wagner A: Robustness and Evolvability in Living Systems (Princeton Studies in Complexity) Princeton University Press; 2005.

10. Forster R, Adami C, Wilke CO: Selection for mutational robustness in finite populations. J Theor Biol 2006, 243: $181-190$.

II. van Nimwegen E, Crutchfield JP, Huynen M: Neutral evolution of mutational robustness. Proc Natl Acad Sci U S A 1999 , 96:9716-9720.

12. Meiklejohn CD, Hartl DL: A single mode of canalization. Trends in Ecology \& Evolution 2002, I 7:468-473.

13. Ancel LW, Fontana W: Plasticity, evolvability, and modularity in RNA. J Exp Zool 2000, 288:242-283.

14. Bornberg-Bauer E, Chan HS: Modeling evolutionary landscapes: mutational stability, topology, and superfunnels in sequence space. Proc Natl Acad Sci U S A 1999, 96: 10689-10694.

15. Bloom JD, Silberg J], Wilke CO, Drummond DA, Adami C, Arnold $\mathrm{FH}$ : Thermodynamic prediction of protein neutrality. Proc Natl Acad Sci U S A 2005, 102:606-6II.

16. Wilke CO, Bloom JD, Drummond DA, Raval A: Predicting the tolerance of proteins to random amino acid substitution. Biophys / 2005, 89:37|4-3720.

17. Rutherford SL, Lindquist S: Hsp90 as a capacitor for morphological evolution. Nature 1998, 396:336-342.

18. Queitsch C, Sangster TA, Lindquist S: Hsp90 as a capacitor of phenotypic variation. Nature 2002, 4I7:618-624.

19. Fares MA, Ruiz-Gonzalez MX, Moya A, Elena SF, Barrio E: Endosymbiotic bacteria: groEL buffers against deleterious mutations. Nature 2002, 417:398.

20. Gibson G, Wagner G: Canalization in evolutionary genetics: a stabilizing theory? Bioessays 2000, 22:372-380.

21. Bonnet E, Wuyts J, Rouze P, Van de PY: Evidence that microRNA precursors, unlike other non-coding RNAs, have lower folding free energies than random sequences. Bioinformatics 2004, 20:291I-2917.

22. Katz L, Burge CB: Widespread selection for local RNA secondary structure in coding regions of bacterial genes. Genome Res 2003, I3:2042-205I.

23. Workman C, Krogh A: No evidence that mRNAs have lower folding free energies than random sequences with the same dinucleotide distribution. Nucleic Acids Res 1999, 27:4816-4822.

24. Rivas E, Eddy SR: Secondary structure alone is generally not statistically significant for the detection of noncoding RNAs. Bioinformatics 2000, 16:583-605.

25. Seffens $W$, Digby D: $\mathbf{m} R \mathbf{N} A s$ have greater negative folding free energies than shuffled or codon choice randomized sequences. Nucleic Acids Res 1999, 27: 1578-I584.

26. Clote P, Ferre F, Kranakis E, Krizanc D: Structural RNA has lower folding energy than random RNA of the same dinucleotide frequency. RNA 2005, I I:578-591.

27. Scharloo W: Canalization: genetic and developmental aspects. Annu Rev Ecol Syst 1991, 22:65-93.

28. Elena SF, Lenski RE: Epistasis between new mutations and genetic background and a test of genetic canalization. Evolution Int J Org Evolution 200I, 55: I746- 1752.

29. Wagner A, Stadler PF: Viral RNA and evolved mutational robustness. J Exp Zool 1999, 285: I |9-127.

30. Zeng $Y$, Cullen BR: Sequence requirements for micro RNA processing and function in human cells. RNA 2003, 9: I | $2-123$.

31. Lee Y, Ahn C, Han J, Choi H, Kim J, Yim J, Lee J, Provost P, Radmark O, Kim S, Kim VN: The nuclear RNase III Drosha initiates microRNA processing. Nature 2003, 425:4I5-4I9.

32. Zeng $Y$, Wagner EJ, Cullen BR: Both natural and designed micro RNAs can inhibit the expression of cognate mRNAs when expressed in human cells. Mol Cell 2002, 9:1327-I333.

33. Zeng Y, Cullen BR: Structural requirements for pre-microRNA binding and nuclear export by Exportin 5. Nucleic Acids Res 2004, 32:4776-4785.

34. Hornstein E, Shomron N: Canalization of development by microRNAs. Nat Genet 2006, 38 Suppl:S20-S24. 
35. Bartel DP: MicroRNAs: genomics, biogenesis, mechanism, and function. Cell 2004, I 16:28I-297.

36. Lau NC, Lim LP, Weinstein EG, Bartel DP: An abundant class of tiny RNAs with probable regulatory roles in Caenorhabditis elegans. Science 200I, 294:858-862.

37. Lagos-Quintana M, Rauhut R, Lendeckel W, Tuschl T: Identification of novel genes coding for small expressed RNAs. Science 200I, 294:853-858.

38. Lee RC, Ambros V: An extensive class of small RNAs in Caenorhabditis elegans. Science 200I, 294:862-864.

39. Brennecke J, Stark A, Russell RB, Cohen SM: Principles of microRNA-target recognition. PLoS Biol 2005, 3:e85.

40. Farh KK: The widespread impact of mammalian MicroRNAs on mRNA repression and evolution. Science 2005, 310:1817-182|.

41. Lewis BP, Burge CB, Bartel DP: Conserved seed pairing, often flanked by adenosines, indicates that thousands of human genes are microRNA targets. Cell 2005, 120:15-20.

42. Xie $X$ : Systematic discovery of regulatory motifs in human promoters and 3[prime] UTRs by comparison of severa mammals. Nature 2005, 434:338-345.

43. Stark A, Brennecke J, Bushati N, Russell RB, Cohen SM: Animal MicroRNAs confer robustness to gene expression and have a significant impact on 3[prime]UTR evolution. Cell 2005, I 23: I I33-I I 46.

44. Grun D, Wang YL, Langenberger D, Gunsalus KC, Rajewsky N: microRNA target predictions across seven Drosophila species and comparison to mammalian targets. PLoS Comput Biol 2005, I:el3.

45. Hutvagner G, McLachlan J, Pasquinelli AE, Balint E, Tuschl T, Zamore PD: A cellular function for the RNA-interference enzyme Dicer in the maturation of the let-7 small temporal RNA. Science 200I, 293:834-838.

46. Lee RC, Feinbaum RL, Ambros V: The C. elegans heterochronic gene lin-4 encodes small RNAs with antisense complementarity to lin-14. Cell 1993, 75:843-854.

47. Ketting RF, Fischer SE, Bernstein E, Sijen T, Hannon GJ, Plasterk RH Dicer functions in RNA interference and in synthesis of small RNA involved in developmental timing in C. elegans. Genes Dev 200I, I 5:2654-2659.

48. Lund E, Guttinger S, Calado A, Dahlberg JE, Kutay U: Nuclear export of microRNA precursors. Science 2004, 303:95-98.

49. Krol J, Sobczak K, Wilczynska U, Drath M, Jasinska A, Kaczynska D, Krzyzosiak W]: Structural features of microRNA (miRNA) precursors and their relevance to miRNA biogenesis and small interfering RNA/short hairpin RNA design. J Biol Chem 2004, 279:42230-42239.

50. Krol J, Krzyzosiak W]: Structural aspects of microRNA biogenesis. IUBMB Life 2004, 56:95-100.

5I. Berezikov E, Guryev V, van de BJ, Wienholds E, Plasterk RH, Cuppen E: Phylogenetic shadowing and computational identification of human microRNA genes. Cell 2005, I20:2I-24.

52. Zuker M, Stiegler P: Optimal computer folding of large RNA sequences using thermodynamics and auxiliary information. Nucleic Acids Res 1981, 9:133-148.

53. Hofacker IL, Fontana W, Stadler PF, Bonhoeffer LS, Tacker M, Schuster P: Fast folding and comparison of RNA secondary structures. Monatshefte fur Chemie / Chemical Monthly 1994, I 25: I 67- I 88

54. Shu W, Bo X, Zheng Z, Wang S: RSRE: RNA structural robustness evaluator. Nucleic Acids Res 2007, 35:W3 I 4-W319.

55. Wuchty S, Fontana W, Hofacker IL, Schuster P: Complete suboptimal folding of RNA and the stability of secondary structures. Biopolymers 1999, 49:145-165.

56. Pang KC, Frith MC, Mattick JS: Rapid evolution of noncoding RNAs: lack of conservation does not mean lack of function. Trends Genet 2006, 22: I-5.

57. Borenstein E, Ruppin E: Direct evolution of genetic robustness in microRNA. Proc Natl Acad Sci U S A 2006, 103:6593-6598.

58. Hermisson J, Wagner GP: Evolution of phenotypic robustness. In Robust Design: A Repertoire from Biology, Ecology, and Engineering Edited by: Jen E. Oxford University Press; 2004:47-70.

59. Bartel DP, Chen CZ: Micromanagers of gene expression: the potentially widespread influence of metazoan microRNAs. Nat Rev Genet 2004, 5:396-400.

60. Berezikov E, Cuppen E, Plasterk RH: Approaches to microRNA discovery. Nat Genet 2006, 38 Suppl:S2-S7.
6I. Kim VN, Nam JW: Genomics of microRNA. Trends Genet 2006, 22:165-173.

62. Bentwich I, Avniel A, Karov Y, Aharonov R, Gilad S, Barad O, Barzilai A, Einat P, Einav U, Meiri E, Sharon E, Spector Y, Bentwich Z: Identification of hundreds of conserved and nonconserved human microRNAs. Nat Genet 2005, 37:766-770.

63. Tuerk C, Gold L: Systematic evolution of ligands by exponential enrichment: RNA ligands to bacteriophage T4 DNA polymerase. Science 1990, 249:505-510.

64. Ellington $A D$, Szostak JW: In vitro selection of RNA molecules that bind specific ligands. Nature 1990, 346:8I8-822.

65. Griffiths-Jones S: The microRNA Registry. Nucleic Acids Res 2004 32:DI09-DIII.

66. Griffiths-Jones S, Grocock RJ, van Dongen S, Bateman A, Enright AJ: miRBase: microRNA sequences, targets and gene nomenclature. Nucleic Acids Res 2006, 34:D I 40-DI 44

67. Fontana W, Konings DA, Stadler PF, Schuster P: Statistics of RNA secondary structures. Biopolymers 1993, 33: | 389-| 404.

68. Shapiro BA: An algorithm for comparing multiple RNA secondary structures. Comput Appl Biosci 1988, 4:387-393.

69. Shapiro BA, Zhang KZ: Comparing multiple RNA secondary structures using tree comparisons. Comput Appl Biosci 1990 6:309-318.

70. Shu W, Bo X, Liu R, Zhao D, Zheng Z, Wang S: RDMAS: a web server for RNA deleterious mutation analysis. $B M C$ Bioinformatics 2006, 7:404.

7I. Hogeweg P, Hesper B: Energy directed folding of RNA sequences. Nucl Acids Res 1984, I 2:67-74.

72. Freyhult E, Gardner PP, Moulton V: A comparison of RNA folding measures. BMC Bioinformatics 2005, 6:24I.

73. Benjamini Y, Hochberg Y: Controlling the False Discovery Rate: A Practical and Powerful Approach to Multiple Testing. J Roy Statist Soc Ser B 1995, 57:289-300.

Publish with BioMed Central and every scientist can read your work free of charge

"BioMed Central will be the most significant development for disseminating the results of biomedical research in our lifetime. "

Sir Paul Nurse, Cancer Research UK

Your research papers will be:

- available free of charge to the entire biomedical community

- peer reviewed and published immediately upon acceptance

- cited in PubMed and archived on PubMed Central

- yours - you keep the copyright

Submit your manuscript here:

http://www.biomedcentral.com/info/publishing_adv.asp
BioMedcentral 